\title{
Prognostic impact of HER2, EGFR, and c-MET status on overall survival of advanced gastric cancer patients
}

\author{
Nozomu Fuse $\cdot$ Yasutoshi Kuboki $\cdot$ Takeshi Kuwata $\cdot$ Tomohiro Nishina $\cdot$ Shigenori Kadowaki \\ Eiji Shinozaki · Nozomu Machida $\cdot$ Satoshi Yuki $\cdot$ Akira Ooki $\cdot$ Shinya Kajiura $\cdot$ Tetsuo Kimura \\ Takeharu Yamanaka $\cdot$ Kohei Shitara $\cdot$ Akiko Kawano Nagatsuma $\cdot$ Takayuki Yoshino • \\ Atsushi Ochiai · Atsushi Ohtsu
}

Received: 24 September 2014/ Accepted: 25 January 2015/Published online: 15 February 2015

(c) The International Gastric Cancer Association and The Japanese Gastric Cancer Association 2015

\begin{abstract}
Background This study was conducted to investigate whether human epidermal growth factor receptor 2 (HER2) status, epidermal growth factor receptor (EGFR) status, and c-MET status are independent prognostic factors for advanced gastric cancer patients who received standard chemotherapy.

Method Unresectable or recurrent gastric or gastroesophageal junction cancer patients with histologically confirmed adenocarcinoma treated with S-1 plus cisplatin as first-line chemotherapy were eligible. Formalin-fixed paraffin-embedded tumor samples were examined for HER2, EGFR, and c-MET status using immunohistochemistry
\end{abstract}

Electronic supplementary material The online version of this article (doi:10.1007/s10120-015-0471-6) contains supplementary material, which is available to authorized users.

N. Fuse $(\bowtie) \cdot$ Y. Kuboki $\cdot$ K. Shitara $\cdot$ T. Yoshino Department of Gastrointestinal Oncology, National Cancer Center Hospital East, 6-5-1 Kashiwanoha, Kashiwa, Chiba 277-8577, Japan

e-mail: nofuse@east.ncc.go.jp

T. Kuwata

Department of Pathology and Clinical Laboratories, National Cancer Center Hospital East, Kashiwa, Japan

T. Kuwata $\cdot$ A. K. Nagatsuma $\cdot$ A. Ochiai Research Center for Innovative Oncology, National Cancer Center Hospital East, Kashiwa, Japan

T. Nishina

Department of Gastrointestinal Medical Oncology, Shikoku

Cancer Center, Matsuyama, Japan

S. Kadowaki

Department of Clinical Oncology, Aichi Cancer Center Hospital,

Nagoya, Japan
(IHC). Additionally, gene amplification was examined using fluorescent in situ hybridization (FISH) for HER2. Positivity was defined as an IHC score of $3+$ or an IHC score of $2+l$ FISH positive for HER2, and an IHC score of $2+$ or $3+$ for both EGFR and c-MET.

Results Of the 293 patients from nine institutions, 43 $(15 \%)$ were HER2 positive, $79(27 \%)$ were EGFR positive, and 120 (41\%) were c-MET positive. Ten patients ( $3 \%$ ) showed positive co-expression of HER2, EGFR, and c-MET. After a median follow-up time of 58.4 months with 280 deaths, there was no significant difference in overall survival (OS) in terms of HER2 and EGFR status. However, there was a significant difference in OS between c-MET-positive and c-MET-negative patients [median, 11.9 months vs 14.2 months; hazard ratio, 1.31 (95\% confidence interval, 1.03-1.67); log-rank $P=0.024]$.

\section{E. Shinozaki}

Gastroenterological Internal Medicine, Cancer Institute Hospital of Japanese Foundation for Cancer Research, Tokyo, Japan

N. Machida

Division of Gastrointestinal Oncology, Shizuoka Cancer Center, Nagaizumi, Japan

S. Yuki

Department of Gastroenterology and Hepatology, Hokkaido University Hospital, Sapporo, Japan

A. Ooki

Department of Gastroenterology, Saitama Cancer Center Hospital, Ina, Japan

S. Kajiura

The Third Department of Internal Medicine, University of Toyama, Toyama, Japan 
Multivariate analysis also showed that c-MET positivity was still a prognostic factor for OS [hazard ratio, 1.30 (95\% confidence interval, 1.02-1.67); $P=0.037]$.

Conclusions The study suggested that c-MET-positive status had poor prognostic value. These data could be used as the basis for future clinical trials for targeting agents for advanced gastric cancer patients.

Keywords c-MET - Epidermal growth factor receptor . Gastric cancer · Gastroesophageal junction cancer · Human epidermal growth factor receptor 2

\section{Introduction}

Human epidermal growth factor receptor 2 (HER2) overexpression has been observed in 9-38\% of gastric cancer patients, and occurs more frequently in gastroesophageal junction (GEJ) and intestinal-type tumors [1]. Treatment with the anti-HER2 monoclonal antibody trastuzumab has been proven to achieve improved survival in HER2-positive advanced gastric cancer (AGC) patients; other new agents such as trastuzumab emtansine and pertuzumab are under investigation [2]. Besides HER2, new agents targeting epidermal growth factor receptor (EGFR) and c-MET have been extensively investigated in gastric cancer [3-5].

HER2 status as a prognostic factor for gastric cancer has been intensively investigated [1]. Although some studies have reported that HER2-positive status was a poor prognostic factor $[6,7]$, others have reported that it was a favorable prognostic factor or was not a significant prognostic factor [8-10]. However, HER2 diagnosis in these studies was based on only immunohistochemistry (IHC) without the standardized scoring system for gastric cancer [2]; most studies were conducted in resected gastric cancer patients and included a wide range of stages, from early gastric cancer to AGC. Therefore, the prognostic impact of HER2 status on overall survival (OS) in AGC

\section{T. Kimura}

Department of Gastroenterology and Oncology, The University of Tokushima Graduate School, Tokushima, Japan

T. Yamanaka

Department of Biostatistics, National Cancer Center, Kashiwa, Japan

T. Yamanaka

Department of Biostatistics, Yokohama City University,

Yokohama, Japan

A. Ohtsu

Exploratory Oncology Research and Clinical Trial Center,

National Cancer Center, Kashiwa, Japan patients treated with standard chemotherapy without trastuzumab for first-line treatment remains controversial.

The prognostic impact and clinicopathological features of EGFR and c-MET have also been studied. EGFR overexpression, which was observed in $27-44 \%$ of gastric cancer patients, has been generally reported to be a poor prognostic factor [11-14]. The correlation between EGFR status and clinicopathological characteristics has not been elucidated. It has been reported that EGFR-positive status was frequently associated with the following factors: noncuratively treated gastric cancer [11]; older age, moderately to poorly differentiated histological appearance, and higher-stage disease [12]; and recurrence after curative surgery and higher disease stages [13]. On the whole, c-MET overexpression, which was observed in $22-82 \%$ of gastric cancer patients, has been reported to be associated with poor prognosis [15-21]; however, findings from a few other studies were contradictory [22, 23]. The correlation between c-MET overexpression and clinicopathological features has been reported in patients with disease with differentiated histological appearance [20, 24, 25], lymph node metastasis $[15,16,23,26]$, peritoneal metastasis [1517], liver metastasis [27], and advanced clinical stage [18, 23, 24]. However, these studies investigated patients who underwent resection, and diagnosis of EGFR and c-MET status was not standardized. Therefore, the prognostic impact of EGFR and c-MET status regarding OS in AGC patients remains unclear; this is also the case for HER2.

Most of the previous studies have involved patients whose disease stages differed, and even studies that have investigated AGC have involved patients who received chemotherapy regimens that were not always standard therapy. To investigate prognosis and elucidate the clinicopathological characteristics, it was considered desirable that the target population should be AGC patients who had received standard therapy [28]. Therefore, we enrolled patients who had received S-1 plus cisplatin as first-line therapy. Our study was conducted to investigate whether HER2 status, EGFR status, and c-MET status are independent prognostic factors in AGC patients, and to elucidate the correlation between this expression status and clinicopathological characteristics.

\section{Patients and methods}

\section{Patients}

We retrospectively collected the clinical data and tumor tissue. The eligibility criteria were as follows: (1) histologically confirmed gastric or GEJ (type I-III tumor using the Siewert classification [29]) adenocarcinoma; (2) unresectable or recurrent cancer; (3) treated with S-1 plus 
cisplatin without trastuzumab as first-line chemotherapy between January 2006 and March 2010 [30]; (4) age 20 years or older; (5) Eastern Cooperative Oncology Group performance status (ECOG PS) of 0-2; and (6) available archived tumor sample. Exclusion criteria included patients' refusal of permission for the use of clinical data and tumor tissue samples, and the presence of other active malignancy.

\section{Clinicopathology data}

We retrospectively collected the following clinicopathology data: age, ECOG PS, sex, primary tumor site, disease status, history of gastrectomy and adjuvant chemotherapy, histological appearance, presence of measurable disease categorized using Response Evaluation Criteria in Solid Tumors version 1.0, location of metastatic sites, serum alkaline phosphatase (ALP) value at the baseline of first-line chemotherapy, OS and progressionfree survival (PFS) from the initiation of the first-line chemotherapy, and details of second-line and later-line chemotherapy if available.

\section{HER2, EGFR, and c-MET assay}

Formalin-fixed paraffin-embedded tumor samples from eligible patients were examined for HER2 using IHC and fluorescent in situ hybridization (FISH), and for EGFR and c-MET using IHC.

HER2 IHC analysis was performed at SRL (Tokyo, Japan) using PATHWAY anti-HER2/neu (4B5) rabbit monoclonal primary antibody (Ventana Medical Systems, Tucson, AZ, USA). The intensity of the membrane staining was evaluated according to the HER2 scoring system for gastric cancer as reported previously [2]. Surgical specimen staining patterns were scored as follows: score 0 , no reactivity or membranous reactivity in less than $10 \%$ of tumor cells; score $1+$, faint/barely perceptible membranous reactivity in $10 \%$ or more of tumor cells or reactive only in part of their membrane; score $2+$, weak to moderate complete or basolateral membranous reactivity in $10 \%$ or more of tumor cells; and score $3+$, moderate to strong complete or basolateral membranous reactivity in $10 \%$ or more of tumor cells (Fig. 1a-d). They were scored for biopsy specimen staining patterns if the staining reactivity of each score was identified in a cluster of five or more tumor cells, irrespective of the percentage of tumor cells stained. A pathologist from SRL primarily determined the HER2 IHC score, and T. Kuwata confirmed the results. If there was discrepancy in the HER2 IHC score among pathologists, the final judgment was made after sufficient discussion between T. Kuwata and A. Ochiai. HER2 FISH analysis was performed at SRL using the PathVysion
HER2 DNA probe kit (Vysis, Downers Grove, IL, USA), according to the manufacturer's instructions. When the ratio of HER2 signal to chromosome 17 centromere signal was 2.0 or greater, the gene was considered as amplified (i.e., FISH positive). HER2 positivity was defined as IHC score $2+$ with a positive FISH result or IHC score $3+$ with any FISH result.

Immunohistochemical staining of EGFR and c-MET was performed automatically by means of Ventana BenchMark ${ }^{\circledR}$ ULTRA using CONFIRM anti-EGFR (3C6) primary antibody (Ventana Medical Systems) and CONFIRM anti-total c-MET (SP44) rabbit monoclonal primary antibody (Ventana Medical Systems) at the National Cancer Center Hospital East. The intensity of the membrane staining regarding EGFR was scored as previously reported [25]: score 0 , no reactivity or membranous reactivity in less than $10 \%$ of tumor cells; score $1+$, faint/barely perceptible membranous reactivity in $10 \%$ or more of tumor cells or cells reactive only in part of their membrane; score $2+$, weak to moderate complete or basolateral membranous reactivity in $10 \%$ or more of tumor cells; and score $3+$, moderate to strong complete or basolateral membranous reactivity in $10 \%$ or more of tumor cells (Fig. 1e-h). EGFR positivity was defined as an IHC score of $2+$ or $3+$. The intensity of the membrane staining regarding c-MET was evaluated as previously reported [31]. Staining patterns were scored as follows: score 0 , no reactivity or less than $50 \%$ of tumor cells with any membranous reactivity; score $1+, 50 \%$ or more of tumor cells with weak or higher membranous reactivity but less than $50 \%$ with moderate or higher membranous reactivity; score $2+, 50 \%$ or more of tumor cells with moderate or higher membranous reactivity but less than $50 \%$ with strong membranous reactivity; and score $3+, 50 \%$ or more of tumor cells with strong membranous reactivity (Fig. 1i-1). We defined c-MET positivity as an IHC membrane staining intensity score of $2+$ or $3+$. Y. Kuboki and T. Kuwata determined the EGFR and c-MET IHC score.

\section{Statistical analysis}

The target accrual of this retrospective study was 300 patients, assuming that more than 40 HER2-positive patients would be enrolled when the proportion of HER2-positive patients was approximately $15 \%$. Differences in patient characteristics were assessed using Fisher's exact test. Survival curves were estimated using the Kaplan-Meier method; OS was calculated from the start of S-1 plus cisplatin administration to death or the last follow-up, and PFS was calculated from the start of S-1 plus cisplatin administration to disease progression assessed by each investigator, death, or the last tumor assessment. The logrank test was used to evaluate the difference between two 


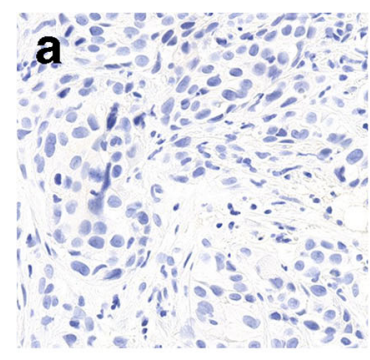

e
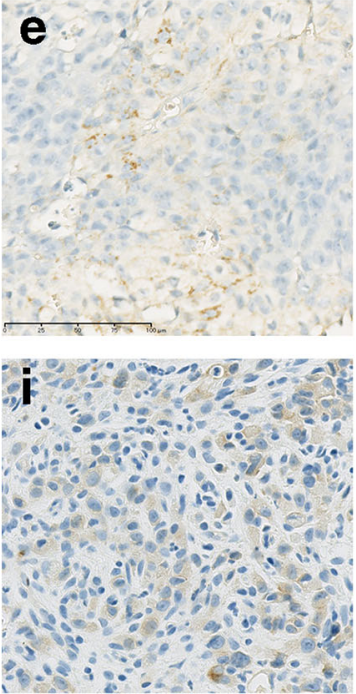

Fig. 1 Representative immunohistochemical staining of human epidermal growth factor receptor 2 (a-d), epidermal growth factor receptor $(\mathbf{e}-\mathbf{h})$, and c-MET (i-l): no membrane staining [immunohistochemistry (IHC) score $0 ; \mathbf{a}, \mathbf{e}, \mathbf{i}]$, faint/barely visible staining

survival curves. Multivariate Cox proportional hazard analysis was performed by the backward elimination with a stay level of 0.10. All $P$ values were reported as two-tailed. Statistical analyses were performed using IBM $^{\circledR}$ SPSS $^{\circledR}$ Statistics version 21 (IBM, Armonk, NY, USA).

Ethical considerations

This study complied with Japanese ethical guidelines for epidemiological research and was approved by the Institutional Review Board and the director of each participating institution.

\section{Results}

Patients and follow-up

We enrolled 293 patients in this study from nine institutions within the planned period. Patient characteristics are detailed in Table 1. The baseline ALP value was not available for nine patients. Of the 293 patients, 213 (73\%) received second-line chemotherapy and 127 (43\%)
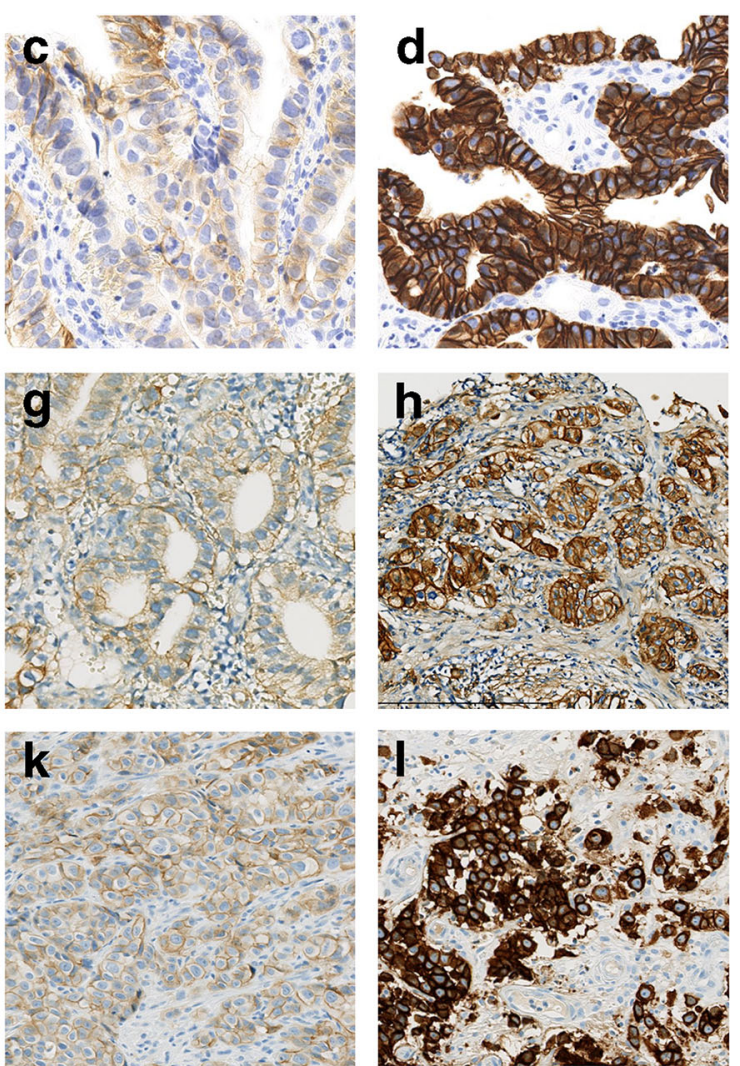

intensity (IHC score $1+; \mathbf{b}, \mathbf{f}, \mathbf{j}$ ), weak to moderate staining intensity (IHC score $2+; \mathbf{c}, \mathbf{g}, \mathbf{k}$ ), and moderate to strong staining intensity (IHC score $3+; \mathbf{d}, \mathbf{h}, \mathbf{l}$ )

received third-line chemotherapy. After a median followup time of 58.4 months, 280 deaths, and 282 cases of progression or death were observed.

Human epidermal growth factor receptor 2

Of the 293 patients, $26(9 \%)$ had an IHC score of $3+, 17$ $(6 \%)$ had an IHC score of $2+$ and were FISH positive, and $43(15 \%)$ were HER2 positive (Table 2). The baseline patient characteristics were significantly different between HER2-positive and HER2-negative patients in terms of histological appearance $(P=0.001)$, presence of measurable disease $(P=0.003)$, number of metastatic sites $(P=0.003)$, presence of liver metastasis $(P=0.003)$, and ALP value $(P<0.001$; Table S1). The location of the primary tumor site did not differ significantly between HER2-positive and HER2-negative patients $(P=0.591$; Table S1). There was no significant difference in PFS \{median, 6.3 months [95\% confidence interval (CI), 5.2-7.3 months] vs 6.4 months [95\% CI, 5.7-7.1 months]; hazard ratio (HR), 1.08 [95\% CI, 0.77-1.50]; log-rank $P=0.662$; Fig. 2a] and OS [median, 11.7 months $(95 \%$ CI, 7.4-16.0 months) vs 13.7 months (95\% CI, 
Table 1 Baseline patient characteristics $(n=293)$

\begin{tabular}{|c|c|c|}
\hline Characteristics & Number & Percentage \\
\hline \multicolumn{3}{|l|}{ Age (years) ${ }^{\mathrm{a}}$} \\
\hline$<65$ & 171 & 58 \\
\hline$\geq 65$ & 122 & 42 \\
\hline \multicolumn{3}{|l|}{ ECOG PS } \\
\hline 0 & 203 & 69 \\
\hline $1-2$ & 90 & 31 \\
\hline \multicolumn{3}{|l|}{ Sex } \\
\hline Male & 201 & 69 \\
\hline Female & 92 & 31 \\
\hline \multicolumn{3}{|l|}{ Primary tumor site } \\
\hline Stomach & 263 & 90 \\
\hline Gastroesophageal junction & 30 & 10 \\
\hline \multicolumn{3}{|l|}{ Disease status } \\
\hline Unresectable & 240 & 82 \\
\hline Recurrent & 53 & 18 \\
\hline \multicolumn{3}{|l|}{ Previous gastrectomy } \\
\hline Yes & 100 & 34 \\
\hline No & 193 & 66 \\
\hline \multicolumn{3}{|c|}{ Previous adjuvant chemotherapy } \\
\hline Yes & 18 & 6 \\
\hline No & 275 & 94 \\
\hline \multicolumn{3}{|l|}{ Histology } \\
\hline Differentiated & 125 & 43 \\
\hline Undifferentiated & 168 & 57 \\
\hline \multicolumn{3}{|l|}{ Measurable disease } \\
\hline Yes & 212 & 72 \\
\hline No & 81 & 28 \\
\hline \multicolumn{3}{|l|}{ Number of metastatic sites } \\
\hline $0-1$ & 146 & 50 \\
\hline$\geq 2$ & 147 & 50 \\
\hline \multicolumn{3}{|l|}{ Metastatic sites } \\
\hline Liver & 101 & 34 \\
\hline Peritoneal & 145 & 49 \\
\hline \multicolumn{3}{|l|}{ Alkaline phosphatase level } \\
\hline Normal & 199 & 68 \\
\hline High & 85 & 29 \\
\hline Not available & 9 & 3 \\
\hline
\end{tabular}

ECOG PS Eastern Cooperative Oncology Group performance status

${ }^{a}$ Median, 62 years, range, 28-82 years

12.4-14.9 months); HR, 1.09 (95\% CI, 0.78-1.51); logrank $P=0.630$; Fig. 2d] between HER2-positive and HER2-negative patients. The proportions of patients who received second-line and third-line chemotherapy were not significantly different between HER2-positive and HER2negative patients $[67 \%$ v. $74 \%$ for second-line chemotherapy $(P=0.459)$ and $35 \%$ vs $45 \%$ for third-line chemotherapy $(P=0.247)]$.
Table 2 Human epidermal growth factor receptor 2 (HER2), epidermal growth factor receptor $(E G F R)$, and c-MET data

\begin{tabular}{lrc}
\hline & Number & Percentage \\
\hline HER2 IHC score & 224 & 76 \\
0 & 13 & 4 \\
$1+$ & 30 & 10 \\
$2+$ & 26 & 9 \\
$3+$ & & \\
HER2 FISH & 54 & 18 \\
HER2/CEN17 $\geq 2.0$ & 230 & 78 \\
HER2/CEN17 $<2.0$ & 9 & 3 \\
No result & & \\
EGFR IHC score & 155 & 53 \\
0 & 59 & 20 \\
$1+$ & 40 & 14 \\
$2+$ & 39 & 13 \\
$3+$ & & \\
c-MET IHC score & 148 & 51 \\
0 & 25 & 92 \\
$1+$ & 95 & 9 \\
$2+$ & 25 & 9 \\
$3+$ & &
\end{tabular}

CEN17 chromosome 17 centromere, FISH fluorescent in situ hybridization, $I H C$ immunohistochemistry

Epidermal growth factor receptor

Of the 293 patients, 79 (27\%) were EGFR positive: 39 (13\%) had an IHC score of 3+, and 40 (14\%) had an IHC score of $2+$ (Table 2). The baseline patient characteristics were significantly different between EGFR-positive and EGFR-negative patients in terms of disease status $(P=0.016$; Table S1). There was no significant difference in PFS [median, 5.8 months (95\% CI, 4.3-7.4 months) vs 6.3 months (95\% CI, 5.8-6.9 months); HR, 1.03 (95\% CI, 0.79-1.34); log-rank $P=0.825$; Fig. 2b] and OS [median, 11.9 months (95\% CI, 10.9-13.0 months) vs 14.2 months (95\% CI, 13.0-15.4 months); HR, 1.12 (95\% CI, 0.86-1.46); log-rank $P=0.401$; Fig. 2e] between EGFR-positive and EGFR-negative patients. The proportions of patients who received second-line and thirdline chemotherapy were not significantly different between EGFR-positive and EGFR-negative patients [71\% vs $73 \%$ for second-line chemotherapy $(P=0.661)$ and $48 \%$ vs $42 \%$ for third-line chemotherapy $(P=0.353)]$.

\section{c-MET}

Of the 293 patients, $25(9 \%)$ had an IHC score of $3+, 95$ (32\%) had an IHC score of $2+$, and $120(41 \%)$ were c-MET-positive (Table 2). The baseline patient 

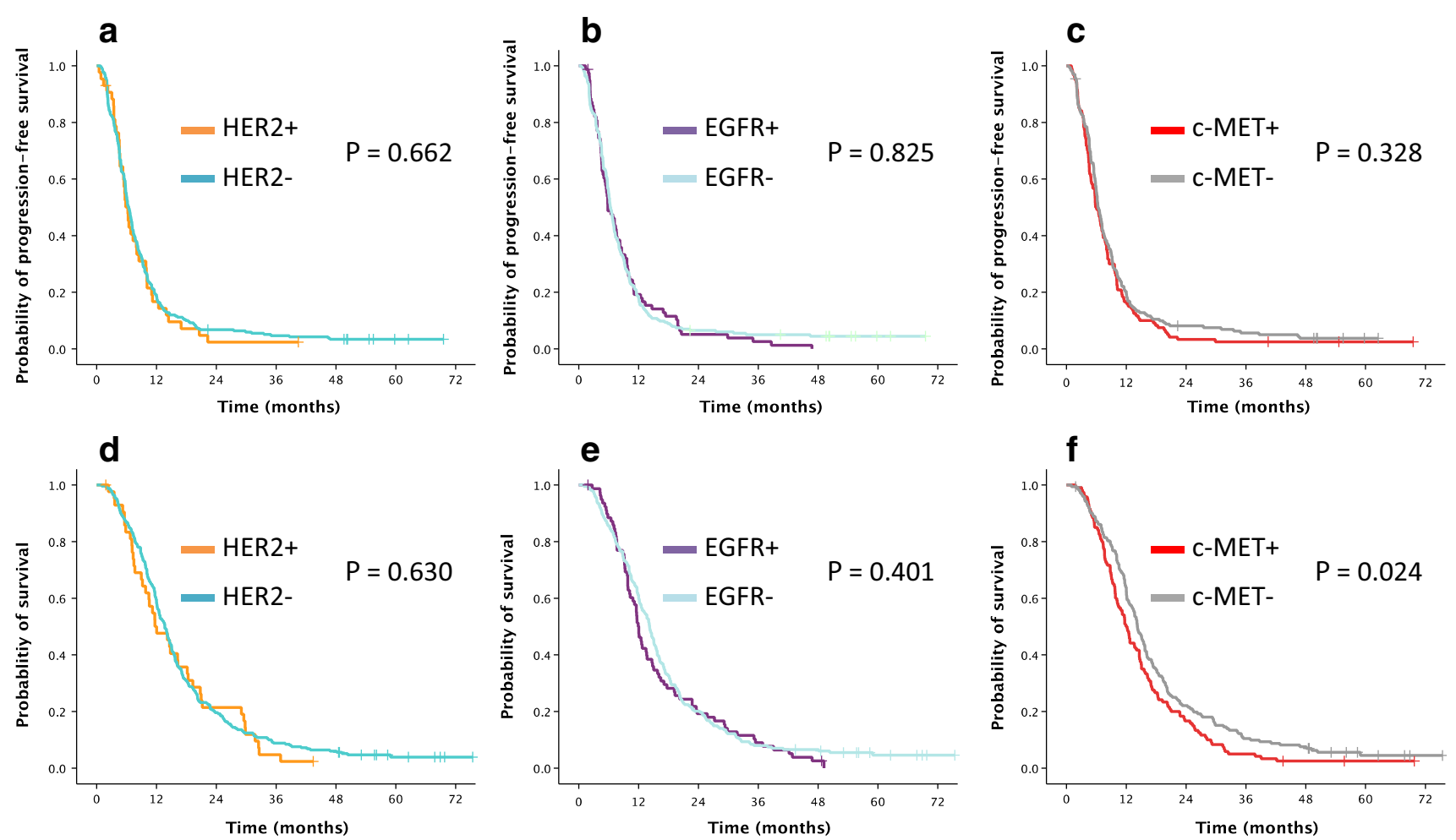

Fig. 2 Progression-free survival and overall survival stratified by human epidermal growth factor receptor 2 (HER2; a, d), epidermal growth factor receptor $(E G F R ; \mathbf{b}, \mathbf{e})$, and c-MET $(\mathbf{c}, \mathbf{f}) . P, \log$-rank $P$ value

characteristics were significantly different between c-METpositive and c-MET-negative patients in terms of histological appearance $(P=0.012)$, liver metastasis $(P=0.034)$, and ALP value $(P=0.047$; Table $\mathrm{S} 1)$. Although there was no significant difference in PFS [median, 5.8 months (95\% CI, 4.9-6.8 months) vs 6.4 months (95\% CI, 5.7-7.1 months); HR, 1.13 (95\% CI, 0.89-1.43); log-rank $P=0.328$; Fig. 2c], there was a significant difference in OS [median, 11.9 months $(95 \%$ CI, 10.7-13.2 months) vs 14.2 months (95\% CI, 12.9-15.5 months); HR, 1.31 (95\% CI, 1.03-1.67); logrank $P=0.024$; Fig. 2f] between c-MET-positive and c-MET-negative patients. The proportions of patients who received second-line and third-line chemotherapy were not significantly different between c-MET-positive and c-MET-negative patients [69 \% vs $75 \%$ for second-line chemotherapy $(P=0.287)$ and $38 \%$ vs $47 \%$ for third-line chemotherapy $(P=0.153)]$.

\section{Co-overexpression of HER2, EGFR, and c-MET}

Co-overexpression status of HER2, EGFR, and c-MET is shown in Fig. 3. Ten patients (3\%) exhibited simultaneous positive status for HER2, EGFR, and c-MET. In the ten simultaneous positive cases, some tumors have three independent areas that exhibited only one protein overexpression (or gene amplification) area, and some tumors have simultaneously two or three protein overexpression (or gene amplification) areas and only one protein overexpression (or gene amplification) area. No specific trends for this heterogeneity and co-overexpression were observed. There was no significant difference in OS among patients with co-overexpression status (data not shown). EGFR positivity between HER2-positive and HER2negative patients was not significantly different $(37 \%$ vs $25 \% ; P=0.135)$. In contrast, c-MET positivity between HER2-positive and HER2-negative patients $(56 \%$ vs $38 \%$ ) and EGFR positivity between c-MET-positive and c-MET-negative patients (37\% vs $20 \%)$ were significantly different $(P=0.043$ and $P=0.002$, respectively).

Multivariate analysis

The multivariate analysis without ALP revealed that c-MET positivity was still a significant prognostic factor for OS [HR, 1.30 (95\% CI, 1.02-1.67); $P=0.037$; Table 3]. As a sensitivity analysis, we conducted a multivariate analysis involving 284 patients, because there was no baseline ALP value for nine patients. It also showed that c-MET positivity was a significant prognostic factor for OS [HR, 1.32 (95 \% CI, 1.02-1.69); $P=0.033$ ]. 
Fig. 3 Co-overexpression status of human epidermal growth factor receptor 2 (HER2), epidermal growth factor receptor $(E G F R)$, and c-MET

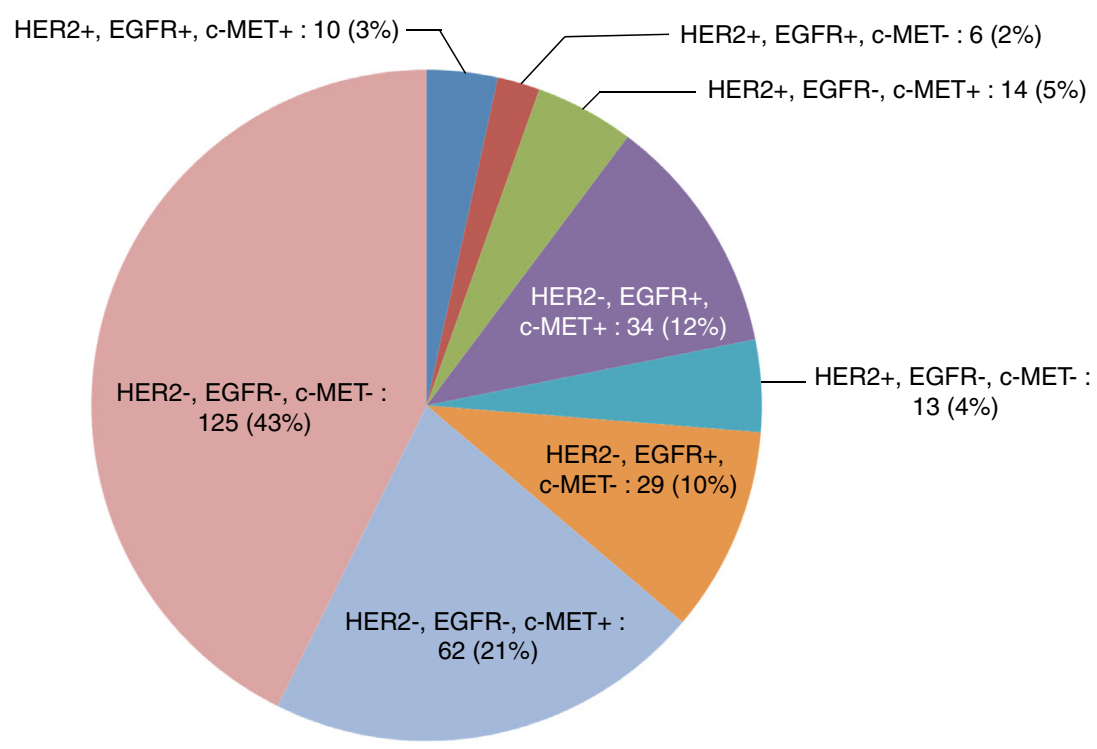

Table 3 Results of multivariate analyses for overall survival $(n=293)$

\begin{tabular}{lllr}
\hline Variable & HR & $95 \%$ CI & \multicolumn{1}{l}{$P$} \\
\hline ECOG PS, 0 & 0.78 & $0.60-1.02$ & 0.067 \\
Primary tumor site, stomach & 1.47 & $0.98-2.21$ & 0.061 \\
Disease status, unresectable & 0.52 & $0.32-0.85$ & 0.009 \\
Previous gastrectomy, yes & 0.32 & $0.21-0.47$ & $\leq 0.001$ \\
Previous adjuvant chemotherapy, yes & 1.78 & $0.99-3.20$ & 0.054 \\
Histological appearance, differentiated & 0.73 & $0.56-0.94$ & 0.014 \\
Measurable disease, yes & 1.32 & $0.95-1.83$ & 0.094 \\
Liver metastasis & 1.56 & $1.16-2.10$ & 0.004 \\
Peritoneal metastasis & 1.27 & $0.96-1.69$ & 0.098 \\
c-MET, positive & 1.30 & $1.02-1.67$ & 0.037 \\
\hline
\end{tabular}

Values in italics indicate statistical significance.

CI confidence interval, ECOG PS Eastern Cooperative Oncology Group performance status, $H R$ hazard ratio

\section{Discussion}

To the best of our knowledge, this is the first study that has investigated co-overexpression of HER2, EGFR, and c-MET in AGC patients who received standard chemotherapy; we found that only c-MET was a significant and independent prognostic factor, which suggests that c-MET would be a good candidate for molecular target agents. There was no significant difference in terms of PFS for S-1 plus cisplatin. Although there were no statistically significant differences in the proportions of patients who received second-line and third-line chemotherapy, fewer c-MET-positive patients received second-line and thirdline chemotherapy; this might have contributed to their poor prognosis. However, we could not reach a definitive conclusion as to the reason for their poor prognosis in this study. We observed c-MET overexpression more frequently in patients with disease with differentiated histological appearance, liver metastasis, and increased ALP levels, which was consistent with the findings of previous studies, except for the ALP levels [20, 24, 27].

Our observation that HER2 was not a prognostic factor in AGC patients was consistent with recent studies that evaluated HER2 using IHC combined with in situ hybridization [14, 32-34]. Although previous studies have reported that HER2 overexpression was more frequent in the GEJ than in the stomach [1], there was no significant difference in the incidence of HER2 positivity between the GEJ and the stomach in our study. The frequency of occurrence of GEJ tumors in our study was relatively small $(10 \%)$ compared with those reported in recent global clinical trials (16-29\%) [4, 35, 36]. Although the exact reason for the discrepancy with other studies remains unknown, our study results were consistent with a recent report concerning Japanese patients: the presence of HER2 overexpression was not influenced by tumor location [37].

Although EGFR has been reported to be a prognostic factor, findings from our study were not consistent with those from previous studies [11-14]. The primary antiEGFR antibody used and the diagnostic criteria were different among the studies. Most importantly, there was a difference in the target populations. Most of the former studies mainly consisted of patients with resectable disease, whereas our study was conducted in patients with unresectable or recurrent disease. Our analysis was consistent with the recent biomarker analysis from the large clinical trial of AGC patients that reported no relationship between the EGFR IHC score and prognosis [4]. 
In the present study, approximately $60 \%$ of AGC patients have overexpression of one or more receptors that can be a target of molecular targeted therapy. Although trastuzumab combined with chemotherapy is a standard first-line treatment for HER2-positive AGC patients, no target-based standard therapy has been established for the remaining AGC patients. For EGFR, although the clinical trials evaluating the anti-EGFR antibodies, such as cetuximab or panitumumab combined with chemotherapy as first-line treatment, failed to show any benefits in nonselected AGC patients, the phase III trial of nimotuzumab as a second-line treatment for EGFR-positive patients is ongoing. For c-MET and its ligand, hepatocyte growth factor, phase III trials of onartuzumab and rilotumumab in AGC patients with c-MET overexpression and a considerable number of phase I trials of c-MET-targeting agents are ongoing [5]. Therefore, a new treatment strategy for patients with simultaneous positivity for EGFR or c-MET and HER2 is required [25].

Our study had several limitations. First, the diagnostic criteria for EGFR and c-MET status were tentative and not standardized. Second, we analyzed EGFR and c-MET only for protein overexpression using IHC, and not for gene amplification. In terms of clinical utility, standardized methods and diagnostic criteria should be established on the basis of the ability to evaluate pharmacological response to therapeutic intervention. Although an exploratory analysis of the clinical utility of EGFR and c-MET status has been conducted in recent clinical trials $[4,5]$, their diagnostic criteria have not been validated. Therefore, diagnostic criteria regarding EGFR and c-MET should be investigated in the ongoing or future prospective clinical trials. Since recent clinical trials often use the diagnostic criteria with IHC for gastric cancer patient enrichment, the correlation between IHC overexpression and the affinity of each drug, and tumor heterogeneity may define the success of clinical development of each agent.

\section{Conclusions}

Findings from the present study suggest that HER 2 and EGFR status had no significant prognostic impact in terms of OS in AGC patients treated with conventional chemotherapy as a first-line treatment. In addition, c-METpositive AGC patients had a poorer prognosis than c-METnegative patients. These data could be used as the basis for future clinical trials for targeting agents in the treatment of AGC patients.

Acknowledgments This study was supported in part by the National Cancer Center Research and Development Fund (21-S4-5 and 23-A-2). We sincerely thank M. Ozawa for data management.
Conflict of interest N. Fuse has received honoraria from Chugai and Taiho, and research funding from Chugai, Taiho, and Daiichi Sankyo. S. Yuki has received honoraria from Chugai, Taiho, and Takeda. T. Yamanaka has received honoraria from Chugai, Taiho, Takeda, and Bristol-Myers Squibb. T. Yoshino has had a consultancy or advisory role for Takeda, and has received honoraria and research funding from Bayer, Daiichi Sankyo, ImClone Systems, and Taiho. A. Ohtsu has received honoraria from Chugai, Roche, and Taiho. All remaining authors declare that they have no conflict of interest.

\section{References}

1. Gravalos C, Jimeno A. HER2 in gastric cancer: a new prognostic factor and a novel therapeutic target. Ann Oncol. 2008;19:1523-9.

2. Bang YJ, Van Cutsem E, Feyereislova A, Chung HC, Shen L, Sawaki A, et al. Trastuzumab in combination with chemotherapy versus chemotherapy alone for treatment of HER2-positive advanced gastric or gastro-oesophageal junction cancer (ToGA): a phase 3, open-label, randomised controlled trial. Lancet. 2010;376:687-97.

3. Waddell T, Chau I, Cunningham D, Gonzalez D, Frances A, Okines C, et al. Epirubicin, oxaliplatin, and capecitabine with or without panitumumab for patients with previously untreated advanced oesophagogastric cancer (REAL3): a randomised, openlabel phase 3 trial. Lancet Oncol. 2013;14:481-9.

4. Lordick F, Kang YK, Chung HC, Salman P, Oh SC, Bodoky G, et al. Capecitabine and cisplatin with or without cetuximab for patients with previously untreated advanced gastric cancer (EXPAND): a randomised, open-label phase 3 trial. Lancet Oncol. 2013;14:490-9.

5. Iveson T, Donehower RC, Davidenko I, Tjulandin S, Deptala A, Harrison $\mathrm{M}$, et al. Rilotumumab in combination with epirubicin, cisplatin, and capecitabine as first-line treatment for gastric or oesophagogastric junction adenocarcinoma: an open-label, dose de-escalation phase $1 \mathrm{~b}$ study and a double-blind, randomised phase 2 study. Lancet Oncol. 2014;15:1007-18.

6. Yonemura Y, Ninomiya I, Yamaguchi A, Fushida S, Kimura H, Ohoyama $\mathrm{S}$, et al. Evaluation of immunoreactivity for erbB-2 protein as a marker of poor short term prognosis in gastric cancer. Cancer Res. 1991;51:1034-8.

7. Allgayer H, Babic R, Gruetzner KU, Tarabichi A, Schildberg FW, Heiss MM. c-erbB-2 is of independent prognostic relevance in gastric cancer and is associated with the expression of tumorassociated protease systems. J Clin Oncol. 2000;18:2201-9.

8. Jain S, Filipe MI, Gullick WJ, Linehan J, Morris RW. c-erbB-2 proto-oncogene expression and its relationship to survival in gastric carcinoma: an immunohistochemical study on archival material. Int J Cancer. 1991;48:668-71.

9. Matsubara J, Yamada Y, Hirashima Y, Takahari D, Okita NT, Kato K, et al. Impact of insulin-like growth factor type 1 receptor, epidermal growth factor receptor, and HER2 expressions on outcomes of patients with gastric cancer. Clin Cancer Res. 2008; 14:3022-9.

10. Grabsch H, Sivakumar S, Gray S, Gabbert HE, Muller W. HER2 expression in gastric cancer: rare, heterogeneous and of no prognostic value-conclusions from 924 cases of two independent series. Cell Oncol. 2010;32:57-65.

11. Galizia G, Lieto E, Orditura M, Castellano P, Mura AL, Imperatore $\mathrm{V}$, et al. Epidermal growth factor receptor (EGFR) expression is associated with a worse prognosis in gastric cancer patients undergoing curative surgery. World J Surg. 2007;31:1458-68. 
12. Kim MA, Lee HS, Lee HE, Jeon YK, Yang HK, Kim WH. EGFR in gastric carcinomas: prognostic significance of protein overexpression and high gene copy number. Histopathology. 2008;52:738-46.

13. Hayashi M, Inokuchi $\mathrm{M}$, Takagi $\mathrm{Y}$, Yamada $\mathrm{H}$, Kojima $\mathrm{K}, \mathrm{Ku}-$ magai $J$, et al. High expression of HER3 is associated with a decreased survival in gastric cancer. Clin Cancer Res. 2008;14:7843-9.

14. Terashima M, Kitada K, Ochiai A, Ichikawa W, Kurahashi I, Sakuramoto $\mathrm{S}$, et al. Impact of expression of human epidermal growth factor receptors EGFR and ERBB2 on survival in stage II/ III gastric cancer. Clin Cancer Res. 2012;18:5992-6000.

15. Yonemura Y, Kaji M, Hirono Y, Fushida S, Tsugawa K, Fujimura T, et al. Correlation between overexpression of c-met gene and the progression of gastric cancer. Int J Oncol. 1996;8:555-60.

16. Taniguchi K, Yonemura Y, Nojima N, Hirono Y, Fushida S, Fujimura $\mathrm{T}$, et al. The relation between the growth patterns of gastric carcinoma and the expression of hepatocyte growth factor receptor (c-met), autocrine motility factor receptor, and urokinase-type plasminogen activator receptor. Cancer. 1998;82:2112-22.

17. Tsugawa K, Yonemura Y, Hirono Y, Fushida S, Kaji M, Miwa K, et al. Amplification of the c-met, c-erbB-2 and epidermal growth factor receptor gene in human gastric cancers: correlation to clinical features. Oncology. 1998;55:475-81.

18. Huang TJ, Wang JY, Lin SR, Lian ST, Hsieh JS. Overexpression of the c-met protooncogene in human gastric carcinoma-correlation to clinical features. Acta Oncol. 2001;40:638-43.

19. Lee HE, Kim MA, Lee HS, Jung EJ, Yang HK, Lee BL, et al. MET in gastric carcinomas: comparison between protein expression and gene copy number and impact on clinical outcome. Br J Cancer. 2012;107:325-33.

20. Ha SY, Lee J, Kang SY, Do IG, Ahn S, Park JO, et al. MET overexpression assessed by new interpretation method predicts gene amplification and poor survival in advanced gastric carcinomas. Mod Pathol. 2013;26:1632-41.

21. An X, Wang F, Shao Q, Wang FH, Wang ZQ, Wang ZQ, et al. MET amplification is not rare and predicts unfavorable clinical outcomes in patients with recurrent/metastatic gastric cancer after chemotherapy. Cancer. 2014;120:675-82.

22. Retterspitz MF, Monig SP, Schreckenberg S, Schneider PM, Holscher AH, Dienes HP, et al. Expression of $\beta$-catenin, MUC1 and c-met in diffuse-type gastric carcinomas: correlations with tumour progression and prognosis. Anticancer Res. 2010;30:4635-41.

23. Li Y, Chen CQ, He YL, Cai SR, Yang DJ, He WL, et al. Abnormal expression of E-cadherin in tumor cells is associated with poor prognosis of gastric carcinoma. J Surg Oncol. 2012;106:304-10.

24. Sotoudeh K, Hashemi F, Madjd Z, Sadeghipour A, Molanaei S, Kalantary E. The clinicopathologic association of c-MET overexpression in Iranian gastric carcinomas; an immunohistochemical study of tissue microarrays. Diagn Pathol. 2012;7:57.

25. Nagatsuma AK, Aizawa M, Kuwata T, Doi T, Ohtsu A, Fujii H, et al. Expression profiles of HER2, EGFR, MET and FGFR2 in a large cohort of patients with gastric adenocarcinoma. Gastric Cancer. 2014. doi:10.1007/s10120-014-0360-4.

26. Nakajima M, Sawada H, Yamada Y, Watanabe A, Tatsumi M, Yamashita $\mathrm{J}$, et al. The prognostic significance of amplification and overexpression of c-met and c-erb B-2 in human gastric carcinomas. Cancer. 1999;85:1894-902.

27. Amemiya H, Kono K, Itakura J, Tang RF, Takahashi A, An FQ, et al. c-Met expression in gastric cancer with liver metastasis. Oncology. 2002;63:286-96.

28. Mandrekar SJ, Sargent DJ. Clinical trial designs for predictive biomarker validation: theoretical considerations and practical challenges. J Clin Oncol. 2009;27:4027-34.

29. Siewert JR, Stein HJ. Classification of adenocarcinoma of the oesophagogastric junction. Br J Surg. 1998;85:1457-9.

30. Koizumi W, Narahara H, Hara T, Takagane A, Akiya T, Takagi $\mathrm{M}$, et al. S-1 plus cisplatin versus S-1 alone for first-line treatment of advanced gastric cancer (SPIRITS trial): a phase III trial. Lancet Oncol. 2008;9:215-21.

31. Spigel DR, Ervin TJ, Ramlau RA, Daniel DB, Goldschmidt JH Jr, Blumenschein GR Jr, et al. Randomized phase II trial of Onartuzumab in combination with erlotinib in patients with advanced non-small-cell lung cancer. J Clin Oncol. 2013;31:4105-14.

32. Okines AF, Thompson LC, Cunningham D, Wotherspoon A, Reis-Filho JS, Langley RE, et al. Effect of HER2 on prognosis and benefit from peri-operative chemotherapy in early oesophago-gastric adenocarcinoma in the MAGIC trial. Ann Oncol. 2013;24:1253-61.

33. Janjigian YY, Werner D, Pauligk C, Steinmetz K, Kelsen DP, Jager E, et al. Prognosis of metastatic gastric and gastroesophageal junction cancer by HER2 status: a European and USA International collaborative analysis. Ann Oncol. 2012;23:2656-62.

34. Shitara K, Yatabe Y, Matsuo K, Sugano M, Kondo C, Takahari $\mathrm{D}$, et al. Prognosis of patients with advanced gastric cancer by HER2 status and trastuzumab treatment. Gastric Cancer. 2013;16:261-7.

35. Ohtsu A, Ajani JA, Bai YX, Bang YJ, Chung HC, Pan HM, et al. Everolimus for previously treated advanced gastric cancer: results of the randomized, double-blind, phase III GRANITE-1 study. J Clin Oncol. 2013;31:3935-43.

36. Wilke H, Van Cutsem E, Oh SC, Bodoky G, Shimada Y, Hironaka S, et al. RAINBOW: a global, phase III, randomized, double-blind study of ramucirumab plus paclitaxel versus placebo plus paclitaxel in the treatment of metastatic gastroesophageal junction (GEJ) and gastric adenocarcinoma following disease progression on first-line platinum- and fluoropyrimidine-containing combination therapy rainbow IMCL CP12-0922 (I4T-IEJVBE). J Clin Oncol. 2014;32(3 Suppl):LBA7.

37. Aizawa M, Nagatsuma AK, Kitada K, Kuwata T, Fujii S, Kinoshita T, et al. Evaluation of HER2-based biology in 1,006 cases of gastric cancer in a Japanese population. Gastric Cancer. 2014;17:34-42. 